\title{
Multi-Clock Path Analysis Using Propositional Satisfiability
}

\author{
Kazuhiro Nakamura Shinji Maruoka Shinji Kimura Katsumasa Watanabe \\ Graduate School of Information Science, \\ Nara Institute of Science and Technology \\ 8916-5 Takayama, Ikoma, Nara, 630-0101 JAPAN \\ Tel: $+81-743-72-5306$ \\ Fax: +81-743-72-5309 \\ e-mail: \{kazuhi-n,sinji-m,kimura,watanabe $\} @$ is.aist-nara.ac.jp
}

\begin{abstract}
We present a satisfiability based multi-clock path analysis method. The method uses propositional satisfiability (SAT) in the detection of multi-clock paths. We show a method to reduce the multi-clock path detection problems to SAT problems. We also show heuristics on the conversion from multi-level circuits into CNF formulae. We have applied our method to ISCAS89 benchmarks and other sample circuits. Experimental results show the improvement on the manipulatable size of circuits by using SAT.
\end{abstract}

\section{INTRODUCTION}

The clock frequency of a sequential logic circuit is decided based on the maximum delay time of the combinational parts in the circuit. The precise estimation of the maximum delay time is important in deciding the proper clock frequency[14].

In logic circuits, there may exist paths where the signal propagation can use 2 or more clock cycles, which are called multiclock paths(or multi-cycle paths)[4],[2],[3]. In other words, each path in the circuit has the number of allowable clock cycles to propagate signals, and if the number is 2 or more, then the delay time of the path can be greater than the clock period. Hence multi-clock paths should be considered in the decision of clock frequency, timing verification, and logic optimization.

There have been proposed two multi-clock path detection methods[4],[2]. One is based on the analysis of state transition graphs of the controllers of microprocessors[4], the other is based on the symbolic state traversal of finite state machines (FSMs)[2]. Both of them detect multi-clock paths based on state traversal of finite state machines, and can only be applied to rather small circuits.

Propositional satisfiability (SAT) is a method to decide the satisfiability of Boolean formulae, and has been used in logic comparison and test pattern generation[5]. Recently, SAT is applied to the analysis of sequential circuits, such as symbolic model checking verification[1] and timing analysis of combinational circuits[10].

In this paper, we present a SAT-based multi-clock path analysis method. The method generates a propositional formula which is satisfiable if and only if the path is not a multi-clock path, and checks the satisfiability of the formula with a propositional prover. We introduce heuristics on the conversion from multi-level circuits to CNF formulae.

We have implemented our method and tested it on ISCAS89 benchmarks [8] and other sample circuits. Experimental results show the improvement on the manipulatable circuit size.

This paper is organized as follows. In the next section, we show preliminaries. In Section III, we present multi-clock path analysis. In Section IV, we present multi-clock path analysis using propositional satisfiability. In Section V, we present heuristics on the translation from a multi-level circuit into a CNF formula with taking the level of a gate into account. In Section VI, we show the experimental results.

\section{PRELIMINARIES}

In this section, we show definitions of sequential circuits and propositional satisfiability.

\section{A. Finite State Machine(FSM)}

We show a definition of an FSM.

Definition 1 An FSM $M$ is a 6-tuple $\left(S, \Sigma, \Gamma, \delta, \lambda, q_{0}\right)$ where

- $S$ is a finite set of states,

- $\Sigma$ is an input alphabet,

- $\Gamma$ is an output alphabet,

- $\delta: S \times \Sigma \rightarrow S$ is a state transition function,

- $\lambda: S \times \Sigma \rightarrow \Gamma$ is an output function,

- $q_{0}(\in S)$ is the initial state.

The behavior of $M$ with respect to an input sequence $a_{1} a_{2} \ldots a_{n}\left(a_{i} \in \Sigma\right)$ is a sequence of states $q_{0} q_{1} \ldots q_{n}\left(q_{i} \in S\right)$ and a sequence of outputs $o_{1} o_{2} \ldots o_{n}\left(o_{i} \in \Gamma\right)$, where each of the states and the outputs satisfies $q_{i}=\delta\left(q_{i-1}, a_{i}\right)$ and $o_{i}=$ $\lambda\left(q_{i-1}, a_{i}\right)$.

Let $\Sigma^{*}$ be the set of all input sequences over $\Sigma$, and let $\Sigma^{k}$ be a set of input sequences with length $k$. We use $\varepsilon$ as the sequence whose length is 0 .

To represent the behavior of $M$, the domain of $\delta$ is extended, and $\delta^{*}: S \times \Sigma^{*} \rightarrow S$ is introduced.

Definition $2 \delta^{*}: S \times \Sigma^{*} \rightarrow S$ is defined as follows:

- $\delta^{*}(q, \varepsilon)=q$

- $\delta^{*}(q, w a)=\delta\left(\delta^{*}(q, w), a\right),\left(a \in \Sigma, w \in \Sigma^{*}\right)$

The set $R S$ of reachable states from the initial state $q_{0}$ is defined as follows. 


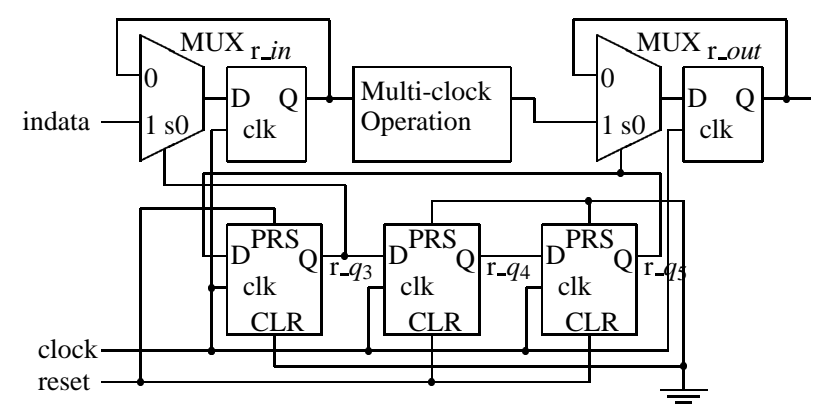

Fig. 1. Example of a multi-clock path.

Definition 3 (Reachable states from the initial state)

$$
R S=\left\{q \mid \exists w \in \Sigma^{*}, q=\delta^{*}\left(q_{0}, w\right)\right\}
$$

Note that a symbol $a_{i} \in \Sigma$ corresponds to a tuple of values of primary inputs of sequential circuits, and a state $q_{j} \in S$ corresponds to a tuple of values of registers in the circuit.

\section{B. Propositional Satisfiability}

Let $x_{i}$ be a propositional variable, and $\bar{x}_{i}$ be the negation of $x_{i}$. A variable and its negation are called literals. The disjunction (OR, $\vee$ ) of literals such as $x_{1} \vee x_{2}$ and $x_{1} \vee \bar{x}_{2} \vee x_{3}$ is a clause. The conjunction of clauses such as $\left(x_{1} \vee x_{2}\right) \wedge\left(x_{1} \vee\right.$ $\left.\bar{x}_{2} \vee x_{3}\right)$ is a conjunctive normal form (CNF) formula. A CNF formula $f$ over $x_{1}, \ldots, x_{l}$ is a CNF formula including only literals $x_{1}, \bar{x}_{1}, x_{2}, \bar{x}_{2}, \ldots, x_{l}, \bar{x}_{l}$.

Let $\left(x_{1}, \ldots, x_{l}\right)$ be a tuple of propositional variables and $f$ be a CNF formula over $x_{1}, \ldots, x_{l}$. We can assign $\mathrm{T}$ (true) or $\mathrm{F}$ (false) for $x_{i}$. If $x_{i}$ is assigned $\mathrm{T}(\mathrm{F})$, then $\bar{x}_{i}$ is assigned $\mathrm{F}(\mathrm{T})$. A clause has value $\mathrm{T}$, if one of literals in the clause is assigned T. A CNF formula has value T, if all clauses have value T. A CNF formula $f$ is satisfiable if $f$ has value $\mathrm{T}$ with some value assignments for variables.

\section{Multi-Clock Path Analysis}

In this section we define multi-clock paths and show a detection method.

\section{A. Multi-Clock Path}

Multi-clock paths are the paths where the propagation of signals can use 2 or more clock cycles. Fig. 1 shows an example of multi-clock paths. The upper part of Fig. 1 shows the data path, and the lower part is the control registers. The initial state of the control registers after the reset signal is $\left(r_{-} q_{3}, r_{-} q_{4}\right.$, $\left.\mathrm{r}_{-} q_{5}\right)=(1,0,0)$, and the state of registers changes as $(0,1,0)$ $\rightarrow(0,0,1) \rightarrow(1,0,0) \rightarrow(0,1,0) \rightarrow \ldots$ synchronized with the clock signal.

At the data path, "r_in" is set to "indata" when $r_{-} q_{3}=1$, and "r_out" is set to the value of the output of "Multi-clock Operation" when $r_{-} q_{5}=1$. Since the state transition is $(1,0,0) \rightarrow$ $(0,1,0) \rightarrow(0,0,1)$, "r_out" is set after 2 clocks with respect to the time when "r_in" is set. Thus we can use 2 clock cycles for the computation of "Multi-clock Operation", and therefore the timing constraint of the path from "r_in" to "r_out" is (the path delay) $\leq 2 \times$ (clock period)

In general, we have the following constraint for each path between registers.

$$
\begin{aligned}
& \text { (the path delay) } \\
& \quad \leq(\text { allowable clock cycles }) \times(\text { clock period })
\end{aligned}
$$

The number of allowable clock cycles can be 2 or more.

Note that the reachable state set plays a key part on the multi-clock path detection. For example, the reachable state set of the above example is $\{(1,0,0),(0,1,0),(0,0,1)\}$, and if all states can be reached, then the signal between $r_{-}$in and r_out should be propagated within 1 cycle when $(1,1,1) \rightarrow(1,1,1)$.

\section{B. Detection Method Based on Symbolic Execution of FSM}

Given a circuit, multi-clock paths are detected by analyzing FSM model of the circuit. At first, we compute the set $R S$ of reachable states from the initial state.

Let $r_{\text {in }}$ and $r_{\text {out }}$ be registers, and there be a path from $r_{\text {in }}$ to $r_{\text {out }}$. If the value of $r_{\text {out }}$ does not change at the next clock of the clock when the value of $r_{\text {in }}$ has just changed, then the propagation of signals can use 2 or more clock cycles. Note that the change of $r_{\text {in }}$ does not contribute to the value of $r_{\text {out }}$. We obtain the following condition specifying the multi-clock property on a path from $r_{\text {in }}$ to $r_{\text {out }}$ :

$$
\begin{aligned}
& \forall q, q^{\prime}, q^{\prime \prime} \in R S, \forall a, a^{\prime} \in \Sigma, \\
& \quad\left[\left(q^{\prime}=\delta(q, a)\right) \wedge\left(q^{\prime \prime}=\delta\left(q^{\prime}, a^{\prime}\right)\right)\right. \\
& \left.\rightarrow\left(\left(q\left(r_{\text {in }}\right) \neq q^{\prime}\left(r_{\text {in }}\right)\right) \rightarrow\left(q^{\prime}\left(r_{\text {out }}\right)=q^{\prime \prime}\left(r_{\text {out }}\right)\right)\right)\right] .
\end{aligned}
$$

Formula (A) denotes that if the value of $r_{\text {in }}$ changes at the state transition from $q$ to $q^{\prime}$, then the value of $r_{\text {out }}$ does not change at the state transition from $q^{\prime}$ to $q^{\prime \prime}$, where $q, q^{\prime}$ and $q^{\prime \prime}$ are reachable from the initial state. Similarly, the condition specifying the path can use 3 or more clock cycles can be obtained by extending Formula (A).

In the following, we focus on Formula (A) specifying the allowable clock cycles to be 2 or more. At present, the symbolic state traversal of the FSM is the best way to compute $R S$, and a BDD-based multi-clock path detection method has been proposed[2]. However, the method is hard to apply to large circuits. Hence, we relax the above condition a little and check the relaxed condition using propositional satisfiability.

\section{Multi-Clock Path Analysis Using SATISFIABILITY}

In this section, we describe an algorithm that detects multiclock paths based on propositional satisfiability.

SAT is not suitable for computing the reachable state set. Thus we abandon the computation and assume that all states are reachable from the initial state. The following formula is used in the multi-clock path analysis instead of Formula (A).

$$
\begin{aligned}
\forall q, q^{\prime}, q^{\prime \prime} & \in S, \forall a, a^{\prime} \in \Sigma \\
{\left[\left(q^{\prime}=\delta(q, a)\right)\right.} & \wedge\left(q^{\prime \prime}=\delta\left(q^{\prime}, a^{\prime}\right)\right) \\
\rightarrow & \left.\left(\left(q\left(r_{\text {in }}\right) \neq q^{\prime}\left(r_{\text {in }}\right)\right) \rightarrow\left(q^{\prime}\left(r_{\text {out }}\right)=q^{\prime \prime}\left(r_{\text {out }}\right)\right)\right)\right] .
\end{aligned}
$$

Since Formula (B) takes into account all states, there is a possibility that several multi-clock paths can not be detected by the analysis as we described in III.A. 


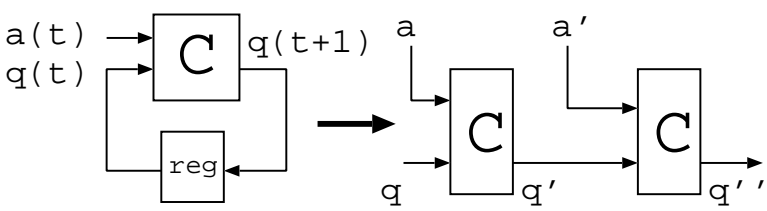

(a) A sequential circuit

(b) A time expansion

Fig. 2. Time expansion of a sequential circuit

\section{A. Time Expansion}

The analysis starts from the time expansion of sequential circuits, where a sequential circuit is translated into a multi-level combinational circuit. Fig. 2 shows the idea of the time expansion: (a) is a sequential circuit and (b) is a time expanded combinational circuit. Since we consider Formula (B), the duplication of a circuit as shown in Fig.2(b) is enough. Symbols in Fig.2(b) correspond to those in Formula (B), where "C" implements the state transition function $\delta$.

\section{B. Propositional Formula for Multi-Clock Path Analysis}

Based on Formula (B), we define a propositional formula $F$ for detecting multi-clock paths.

Let $r_{\text {in }}$ and $r_{\text {out }}$ be the input and output registers to be checked. The following propositional formula $F$ becomes true if the path from $r_{\text {in }}$ to $r_{\text {out }}$ can use 2 clock cycles:

$$
\begin{aligned}
F \triangleq & T\left(a, q, q^{\prime}\right) \wedge T\left(a^{\prime}, q^{\prime}, q^{\prime \prime}\right) \\
& \rightarrow\left(\left(q\left(r_{\text {in }}\right) \not \equiv q^{\prime}\left(r_{\text {in }}\right)\right) \rightarrow\left(q^{\prime}\left(r_{\text {out }}\right) \equiv q^{\prime \prime}\left(r_{\text {out }}\right)\right)\right)
\end{aligned}
$$

where $T\left(a, q, q^{\prime}\right)=1$ iff $q^{\prime}=\delta(q, a)$ and $T\left(a^{\prime}, q^{\prime}, q^{\prime \prime}\right)=1$ iff $q^{\prime \prime}=\delta\left(q^{\prime}, a^{\prime}\right)$, and $q(r)$ denotes the value of a register $r$ under the state $q$. Note that $T\left(a, q, q^{\prime}\right)$ represents a logic formula which is implemented by a multi-level circuit as in Fig.2, where $a, q$ and $q^{\prime}$ are binary coded and propositional variables have been assigned. Also note that $q\left(r_{\text {in }}\right)$ and $q\left(r_{\text {out }}\right)$ are propositional variables corresponding to $r_{\text {in }}$ and $r_{\text {out }}$ in the tuple of the state variables for the state $q$.

If $\bar{F}$ is unsatisfiable, then $F$ is always true for any $q, a$ and $a^{\prime}$, and therefore we can decide that the allowable clock cycle is 2 or more. We translate $\bar{F}$ into CNF formulae, and check the unsatisfiability of $\bar{F}$ using a SAT prover. We can obtain CNF of $\bar{F}$ using a translation rule $a \not \equiv b \Rightarrow(a \vee b) \wedge(\bar{a} \vee \bar{b})$ :

$$
\begin{aligned}
\bar{F}=T(a, & \left.q, q^{\prime}\right) \wedge T\left(a^{\prime}, q^{\prime}, q^{\prime \prime}\right) \\
& \wedge\left(q\left(r_{\text {in }}\right) \vee q^{\prime}\left(r_{\text {in }}\right)\right) \wedge\left(\overline{q\left(r_{\text {in }}\right)} \vee \overline{q^{\prime}\left(r_{\text {in }}\right)}\right) \\
& \wedge\left(q^{\prime}\left(r_{\text {out }}\right) \vee q^{\prime \prime}\left(r_{\text {out }}\right)\right) \wedge\left(\overline{q^{\prime}\left(r_{\text {out }}\right)} \vee \overline{q^{\prime \prime}\left(r_{\text {out }}\right)}\right) .
\end{aligned}
$$

Note that $T\left(a, q, q^{\prime}\right)$ and $T\left(a^{\prime}, q^{\prime}, q^{\prime \prime}\right)$ are the same logic formula with different variables. In the following we discuss a method to generate CNF of $T\left(a, q, q^{\prime}\right)$.

\section{CONVERSION TO CNF FORMULA}

In this section we show a method to translate from multilevel combinational circuits into CNF formulae.

In general, $\mathrm{CNF}$ formulae can be generated from multi-level combinational circuits using the following translation rules: $(a \not \equiv b) \Rightarrow(a \vee b) \wedge(\bar{a} \vee \bar{b}),(a \equiv b) \Rightarrow(a \vee \bar{b}) \wedge(\bar{a} \vee b)$ and $(a \wedge b) \vee c \Rightarrow(a \vee c) \wedge(b \vee c)$. The size of resulting CNF formulae may grow exponentially, and we should introduce heuristic techniques in the conversion. For example, let $f_{1}$ and $f_{2}$ be sub-formulae which are not $\mathrm{CNF}$, and we consider a formula $\left(f_{1} \not \equiv f_{2}\right)$. The formula is translated into $\left(f_{1} \vee f_{2}\right) \wedge\left(\overline{f_{1}} \vee \overline{f_{2}}\right)$ using the above rules, and the size of the formula can be twice. If $f_{2}$ is $f_{21} \wedge f_{22}$, then $f_{1}$ should be duplicated and the size of CNF can be exponential with respect to the size of the original formula in the worst case.

To avoid this problem, we apply a method which introduces new propositional variables and reduces the size of CNF formulae without affecting the satisfiability of formulae[13]. For example, if we introduce new variables $x_{1}$ and $x_{2}$ for $f_{1}$ and $f_{2}$, then we obtain an equivalent formula $\left(x_{1} \not \equiv x_{2}\right) \wedge\left(x_{1} \equiv\right.$ $\left.f_{1}\right) \wedge\left(x_{2} \equiv f_{2}\right)$. In the translation of $x_{1} \equiv f_{1}$ into CNF, the size of CNF formulae does not grow exponentially, since $x_{1}$ is a propositional variable. Note that $x$ is manipulated as a primary input variable.

In [13], new variables are inserted for all logic gates. We adapt the translation method, and improve the method by introducing new heuristic techniques on the insertion of new variables based on the depth of logic gates from primary output and newly introduced variables. In the following, we assume that multi-level combinational logic circuits consist of AND, OR, NOT and $\mathrm{XOR}(\not \equiv)$ gates.

To control the number of introduced variables and the size of generated CNF formulae, we introduce heuristic techniques. Fig. 3 shows the idea. We classify 4 cases as shown in the figure, and introduce 4-level parameters. In Fig.3, "v" denotes logic gates which we focus on, "Var" denote new variables which we introduced. "XOR-level", "OR-level”, "ANDlevel" and "NOT-level" are thresholds that denote the maximum depth from introduced variables. We introduce new variables at the focusing gate $v$ when the number of levels of " $v$ " from the nearest variables exceeds the thresholds. By this, the circuit is divided into sub-circuits with almost the same level. For example, the OR gate in Fig.3(b) is considered. If the level of the OR gate from the nearest variable is over "OR-level" and "pre_v" is not a NOT gate, then we introduce new variables for fan-ins of the OR gate. We show the effect of this method on reducing CNF size in Sect. VI.

Fig.4 shows an algorithm for introducing new variables. The analysis starts from the primary outputs of multi-level logic circuits, and goes backward toward primary inputs based on the depth first search algorithm. At first $l e v$ is set to 1 , and then $l e v$ is incremented for every recursive calls of DepthFirst (). If the value of $l e v$ exceeds the threshold decided by the type of gates, then new variables are inserted for fan-ins by NewVar().

\section{EXPERIMENTAL RESULTS}

We have implemented a SAT-based multi-clock path analyzer in $\mathrm{C}$ language and compared it with a symbolic execution based multi-clock path analyzer using BDD's[2]. We have also implemented BDD-based analyzer without reachability. The SAT-based analyzer reads circuit descriptions in SLIF[16], and produces CNF formulae in DIMACS format[9] for detecting multi-clock paths. The satisfiability of the formula is checked 


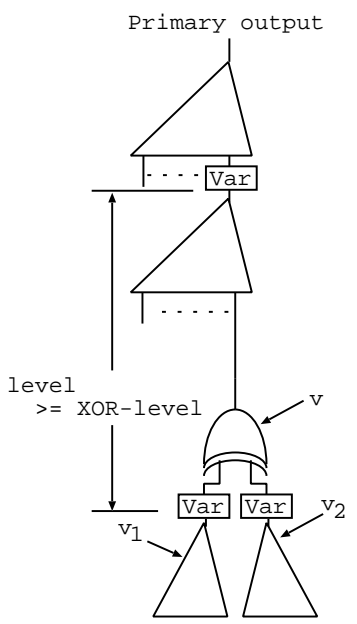

(a) XOR

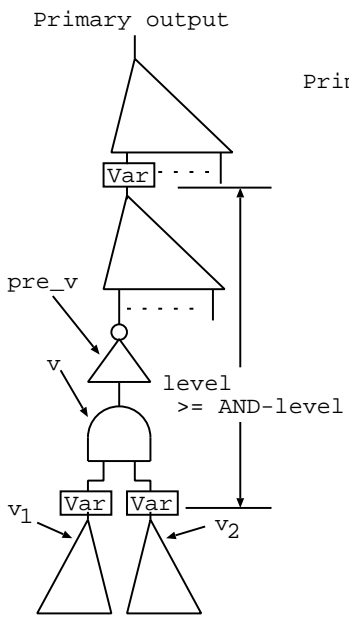

(c) NOT-AND

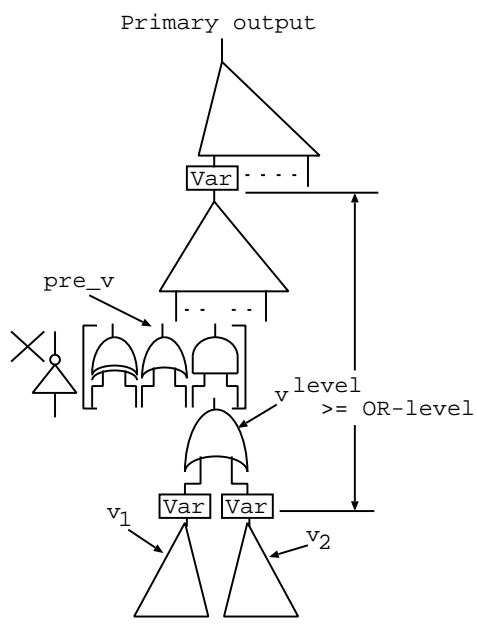

(b) XOR-OR, OR-OR, AND-OR

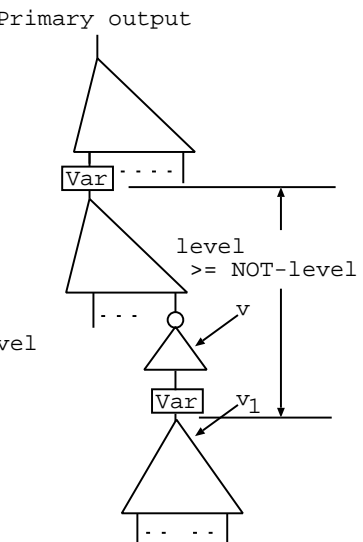

(d) NOT
Fig. 3. Internal variables

with H. Zhang's SATO[6] / J. Silva's GRASP[15]. Both of the SAT provers are based on Davis-Putnam method[7].

We have analyzed the ISCAS89 benchmarks[8] and other sample circuits designed by our laboratory on a PC (CPU Pentium II 500MHz, Main memory 512MB).

\section{A. Application to ISCAS Benchmarks}

We have applied 3 analysis methods to 30 ISCAS89 benchmarks. (1) Method1: BDD-based algorithm which takes into account the reachable state set[2]. (2) Method2: BDD-based algorithm which assumes that all states are reachable, (3) Method3: SAT-based algorithm which assumes that all states are reachable. We used SATO as a SAT prover.

TableI shows the statistics. In the table, "\#In", "\#Reg" and "\#R-pair" are the number of primary inputs, registers and connected pairs of registers in the circuit respectively. "\#Rep", "\#M-pair" and "Time" are the number of repetitions in computing reachable states of the circuit, the number of pairs of registers whose path is a multi-clock path(2-cycle), and the elapsed CPU seconds obtained by the time command respectively. "\#Var", "\#Cl" and "\#Lit" are the maximum number of propositional variables, clauses and literals of the formula which is used in our method. Multi-clock paths on 21 cir-

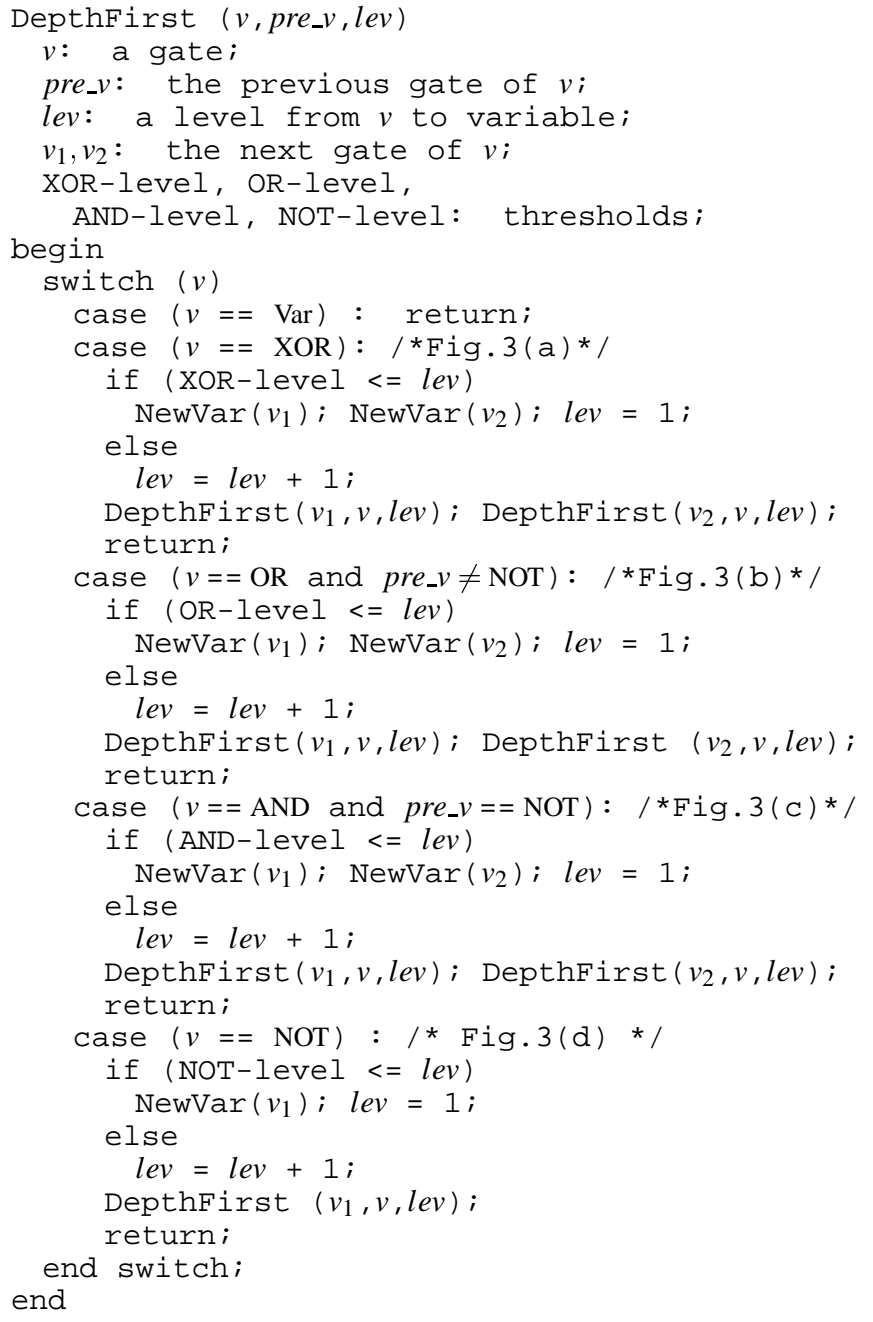

Fig. 4. An algorithm for introducing new variables.

cuits are found by Method1[2]: ex2, ex3, ex4, ex5, ex6, ex7, s208, s298, s344, s349, s382, s386, s420, s444, s510, s526, s526n, s641, s713, s838, s953. Multi-clock paths on only 16 circuits can be found by Method2 and Method3: ex2, ex3, ex4, ex5, ex6, ex7, s298, s344, s349, s382, s386, s444, s510, s526, s526n, s953. Because of the effect of unreachable states, multi-clock paths in s208, s420, s641, s713 and s838 can not be found by Method2 and Method3, and the total number of paths is small on Method2 and Method 3 for detected circuits. The total number of "\#M-pair" in Method1 is 1101, that in Method 2 and Method3 is only 590 and 636. Note that s1423 has memory overflow on BDD-based methods, and Method3 can detect s1423. The elapsed CPU time of Method3 is the smallest.

\section{B. Application to Sample Circuits}

We have applied our SAT-based method and the BDD-based method in [2] to several circuits which are designed in our laboratory: prime 250 (a prime number generator which computes prime numbers less than 250 , about 400 gates), s-div8 (an 8bit sequential divider, about 500 gates), prime999 (a prime number generator which computes prime numbers less than 999, about 400 gates), bezier8 (an 8-bit bezier curve generator, about 900 gates), forsen (an edge detection circuit for im- 
TABLE I

RESULT OF MULTI-CLOCK PATH ANALYSIS OF ISCAS BENCHMARK CIRCUITS.

\begin{tabular}{|c|c|c|c|c|c|c|c|c|c|c|c|c|c|}
\hline \multicolumn{4}{|c|}{ Circuits } & \multicolumn{3}{|c|}{$\begin{array}{c}\text { Method1 } \\
\text { (Reachability, BDD) }\end{array}$} & \multicolumn{2}{|c|}{$\begin{array}{c}\text { Method2 } \\
\text { (BDD) }\end{array}$} & \multicolumn{5}{|c|}{$\begin{array}{c}\text { Method3 } \\
\text { (SAT) }\end{array}$} \\
\hline Name & \#In & \#Reg & \#R-pair & \#Rep & \#M-pair & \begin{tabular}{|l|} 
Time \\
\end{tabular} & \#M-pair & Time & \#M-pair & \#Var & $\mathrm{\# Cl}$ & \#Lit & Time \\
\hline daio & 1 & 4 & $\overline{6}$ & 5 & 0 & 0.8 & $\overline{0}$ & 2.8 & 0 & 18 & 46 & 124 & 0.7 \\
\hline ex2 & 2 & 19 & 342 & 6 & 306 & 1.0 & 238 & 2.1 & 238 & 195 & 1250 & 3554 & 4.6 \\
\hline ex3 & 2 & 10 & 90 & 5 & 80 & 0.8 & 45 & 1.7 & 45 & 102 & 570 & 1626 & 1.2 \\
\hline ex4 & 6 & 14 & 169 & 14 & 135 & 0.9 & 135 & 1.7 & 135 & 116 & 436 & 1172 & 1.5 \\
\hline ex5 & 2 & 9 & 72 & 4 & 48 & 0.8 & 30 & 1.5 & 30 & 93 & 488 & 1380 & 1.0 \\
\hline ex6 & 5 & 9 & 61 & 1 & 61 & 0.5 & $y$ & 1.6 & $\overline{9}$ & 99 & 638 & 2032 & 0.9 \\
\hline ex7 & 2 & 10 & 90 & 5 & 77 & 0.8 & 53 & 1.8 & 53 & 102 & 614 & 1816 & 1.2 \\
\hline s27 & 4 & 3 & 4 & 3 & 0 & 0.6 & 0 & 1.5 & 0 & 23 & 48 & 124 & 0.5 \\
\hline s208 & 11 & 8 & 28 & 17 & 18 & 0.9 & 0 & 1.6 & 0 & 78 & 240 & 700 & 0.5 \\
\hline \begin{tabular}{|l|} 
s298 \\
\end{tabular} & 3 & 14 & 56 & 19 & 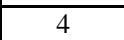 & 0.7 & J & 1.7 & 3 & 112 & 468 & 1438 & 0.9 \\
\hline \begin{tabular}{|l|} 
s344 \\
\end{tabular} & 9 & 15 & 74 & 7 & 1 & 1.9 & 1 & 1.7 & 1 & 155 & 480 & 1296 & 1.3 \\
\hline s349 & 9 & 15 & 74 & 7 & 1 & 1.8 & 1 & 1.6 & 1 & 155 & 484 & 1312 & 1.3 \\
\hline \begin{tabular}{|l|}
$\mathrm{s} 382$ \\
\end{tabular} & 3 & 21 & 131 & 151 & 13 & \begin{tabular}{|l}
11.6 \\
\end{tabular} & 13 & 9.9 & 13 & 175 & 622 & 1738 & 2.0 \\
\hline \begin{tabular}{|l|} 
s386 \\
\end{tabular} & 7 & 6 & 30 & 8 & 4 & 0.9 & 4 & 1.5 & 4 & 78 & 500 & 1824 & 0.9 \\
\hline \begin{tabular}{|l|}
$\mathrm{s} 420$ \\
\end{tabular} & 19 & 16 & 72 & 17 & 62 & 0.9 & 0 & 1.7 & 0 & 158 & 496 & 1420 & 1.1 \\
\hline \begin{tabular}{|l|} 
s444 \\
\end{tabular} & 3 & 21 & 131 & 151 & 13 & \begin{tabular}{|l|}
13.3 \\
\end{tabular} & 13 & 6.3 & 13 & 221 & 844 & 2392 & 2.6 \\
\hline \begin{tabular}{|l|}
$\mathrm{s} 510$ \\
\end{tabular} & 19 & 6 & 30 & 47 & 7 & 0.9 & 2 & 1.6 & 2 & 192 & 814 & 2588 & 1.4 \\
\hline \begin{tabular}{|l|}
$\mathrm{s} 526$ \\
\end{tabular} & 3 & 21 & 123 & 151 & 8 & \begin{tabular}{|l|}
10.3 \\
\end{tabular} & 7 & 20.1 & 7 & 207 & 1030 & 3344 & 2.5 \\
\hline s526n & 3 & 21 & 123 & 151 & 8 & \begin{tabular}{|l}
10.5 \\
\end{tabular} & 7 & \begin{tabular}{|l}
37.7 \\
\end{tabular} & 7 & 203 & 1024 & 3338 & 2.5 \\
\hline \begin{tabular}{|l|} 
s641 \\
\end{tabular} & 35 & 19 & 100 & 7 & 38 & 9.3 & 0 & 5.3 & 0 & 259 & 656 & 1818 & 1.9 \\
\hline \begin{tabular}{|l|} 
s713 \\
\end{tabular} & 35 & 19 & 100 & 7 & 38 & \begin{tabular}{|l|}
10.2 \\
\end{tabular} & 0 & 6.0 & 0 & 293 & 844 & 2344 & 2.6 \\
\hline \begin{tabular}{|l|}
$\mathrm{s} 820$ \\
\end{tabular} & \begin{tabular}{|l|}
18 \\
\end{tabular} & 5 & 20 & 11 & 0 & 0.9 & 0 & 1.5 & 0 & 221 & 1504 & \begin{tabular}{|l}
5768 \\
\end{tabular} & 1.7 \\
\hline \begin{tabular}{|l|}
$\mathrm{s} 832$ \\
\end{tabular} & 18 & 5 & 20 & 11 & 0 & 0.8 & 0 & 1.7 & 0 & 217 & 1538 & 5902 & 1.6 \\
\hline \begin{tabular}{|l|}
$\mathrm{s} 838$ \\
\end{tabular} & 35 & 32 & 160 & 17 & 150 & 1.1 & 0 & 101.1 & 0 & 318 & 1008 & 2860 & 3.0 \\
\hline \begin{tabular}{|l|} 
s953 \\
\end{tabular} & 16 & 29 & 150 & 11 & 29 & 19.9 & 29 & 6.2 & 29 & 469 & 1702 & 4848 & 6.2 \\
\hline s1196 & 14 & 18 & 20 & 3 & 0 & \begin{tabular}{|l|}
253.4 \\
\end{tabular} & 0 & 10.9 & 0 & 318 & 1290 & 3856 & 2.2 \\
\hline s1238 & 14 & 18 & 20 & 3 & 0 & 200.2 & 0 & 12.6 & 0 & 322 & 1338 & 4030 & 2.4 \\
\hline s1423 & 17 & 74 & 1694 & - & Mem $>1 \mathrm{G}$ & - & Mem $>1 \mathrm{G}$ & - & 46 & 654 & 2238 & 6298 & 60.4 \\
\hline s1488 & 8 & 6 & 30 & 23 & 0 & 0.9 & 0 & 1.6 & 0 & 222 & 1576 & 5836 & 2.0 \\
\hline s1494 & 8 & 6 & 30 & 23 & 0 & 0.8 & 0 & 1.7 & 0 & 222 & 1608 & 5980 & 2.0 \\
\hline \multicolumn{4}{|c|}{ Total number of M-pair } & \multicolumn{3}{|c|}{1101} & \multicolumn{2}{|l|}{590} & \multicolumn{5}{|c|}{636} \\
\hline
\end{tabular}

Mem > 1G: we have analyzed on a DEC AlphaServer8400 (CPU alpha21164A 617MHz $\times 10$, Main memory 8GB).

age processing, about 900 gates), pcpu16 (a 16-bit pipelined processor, about 9,000 gates), mul64 (a circuit including a 64bit combinational adder array multiplier, about 47,000 gates), SpchRecog (a HMM-based speech recognition circuit, , about 40,000 gates). They have been designed to include multi-clock paths: those of forsen are to meet the clock constraints, those of SpchRecog are for memory access constraints, and those of others are for experiments.

We have found multi-clock register pairs on all circuits by Method3. TableII shows the statistics. Almost all circuits, BDD-based methods suffer from memory overflow or timeoverflow. We used SATO as a SAT prover if not specified. For only SpchRecog, we should use GRASP to overcome the number of registers more than 1000 .

\section{Effects of Thresholds for Introducing New Variables}

We have tested properties of thresholds which are used to introduce new variables in our algorithm.

TableIII shows statistics of multi-clock path detection of s1423 and mul16. In the table, "Threshold: a, b, c, d" denote "XOR-level", "OR-level", "AND-level" and "NOT-level" in Fig.3. Note that our algorithm with $(1,1,1,2)$ introduces variables for all logic gates. From these tables, the number of literals becomes the smallest when the "Threshold" is $(1,4,4$, 5), and the elapsed CPU time is the shortest when the "Threshold" is $(1,9,9,10)$. The statistics show that the reduction of the size of CNF formula does not always contribute to reducing the time for analysis. Note that the statistics in TableI and TableII are obtained by using $(1,4,4,5)$ because the size of $\mathrm{CNF}$ is more important in the manipulation.

\section{CONCLUSIONS}

In this paper, we have presented multi-clock path analysis method based on propositional satisfiability and shown experimental results.

The method reduces multi-clock path detection problems into SAT problems using the time expansion. The SAT-based algorithm enables us to apply multi-clock path analysis to large circuits that can not be analyzed with the symbolic execution based algorithm.

We have applied our method to ISCAS89 benchmarks and other sample circuits. Experimental results show the improvement on the manipulatable size of circuits by using satisfiability.

The problem is that the SAT-based algorithm can only detect a subset of multi-clock paths detectable by the symbolic 
TABLE II

RESULTS OF MULTI-CLOCK PATH ANALYSIS OF SAMPLE CIRCUITS

\begin{tabular}{|c|c|c|c|c|c|c|c|c|c|c|c|c|c|}
\hline \multicolumn{4}{|c|}{ Circuits } & \multicolumn{3}{|c|}{$\begin{array}{c}\text { Method1 } \\
\text { (Reachability, BDD) }\end{array}$} & \multicolumn{2}{|c|}{$\begin{array}{c}\text { Method2 } \\
\text { (BDD) }\end{array}$} & \multicolumn{5}{|c|}{$\begin{array}{l}\text { Method3 } \\
\text { (SAT) }\end{array}$} \\
\hline Name & \#In & \#Reg & \#R-pair & \#Rep & \#M-pair & Time & \#M-pair & Time & \#M-pair & \#Var & $\mathrm{\# Cl}$ & \#Lit & Time \\
\hline prime 250 & 2 & 35 & 718 & 11959 & 570 & 774.3 & 413 & 116606.6 & 413 & 435 & 1662 & 4768 & 17.4 \\
\hline s-div8 & 10 & 47 & 514 & - & Mem $>1 G$ & - & - & Time $>48 \mathrm{~h}$ & 33 & 557 & 2000 & 5620 & 20.5 \\
\hline prime999 & 2 & 44 & 1219 & - & Mem $>1 \mathrm{G}$ & - & - & Time $>48 \mathrm{~h}$ & 480 & 550 & 2258 & 6498 & 37.1 \\
\hline bezier8 & 34 & 64 & 684 & - & Mem $>1 G$ & - & Mem $>1 G$ & - & 58 & 2121 & 9074 & 27264 & 37.1 \\
\hline forsen & 187 & 255 & 1739 & - & Mem $>1 G$ & - & Mem $>1 G$ & - & 1739 & 2547 & 956 & 24922 & 199.9 \\
\hline pсpu16 & 40 & 779 & 6744 & - & Mem $>1 G$ & - & Mem $>1 G$ & - & 0 & 4675 & 22462 & 71762 & 16292.9 \\
\hline mul64 & 259 & 258 & 12866 & - & Mem $>1 \mathrm{G}$ & - & Mem $>1 G$ & - & 12480 & 39666 & 160114 & 451304 & 97560.6 \\
\hline pchRecog & 37 & 1316 & 134443 & - & Mem $>1 \mathrm{G}$ & - & Mem $>1 G$ & - & 57573 & 22256 & 105252 & 328272 & $547.5 \mathrm{~h}^{\dagger}$ \\
\hline
\end{tabular}

Mem > 1G: we have analyzed on a DEC AlphaServer8400 (CPU alpha21164A 617MHz $\times 10$, Main memory 8GB).

SpchRecog: we have analyzed on 6 Sun Ultra2 workstations (UltraSPARC-IIi 333MHz, Main memory 512MB) in parallel.

$\dagger$ : The total CPU time using GRASP.

TABLE III

THE INFLUENCE OF THE THRESHOLD ON MULTI-CLOCK PATH ANALYSIS.

\begin{tabular}{|c|c|c|c|c|}
\hline \multirow{2}{*}{$\begin{array}{c}\text { Threshold } \\
\mathrm{a}, \mathrm{b}, \mathrm{c}, \mathrm{d}\end{array}$} & \multicolumn{4}{|c|}{$\begin{array}{c}\text { Method3 } \\
\text { (SAT) }\end{array}$} \\
\cline { 2 - 5 } & \#Var & \#Cl & \#Lit & Time \\
\hline $1,14,14,15$ & 414 & 4604 & 25222 & 95.7 \\
\hline $1,9,9,10$ & 460 & 2248 & 7896 & 47.5 \\
\hline $1,4,4,5$ & 654 & 2238 & 6290 & 57.8 \\
\hline $1,1,1,2$ & 1256 & 3232 & 7500 & 83.1 \\
\hline
\end{tabular}

(a) Statistics for $\mathrm{s} 1423$

\begin{tabular}{|c|c|c|c|c|}
\hline \multirow{2}{*}{$\begin{array}{c}\text { Threshold } \\
\text { a, b, c, d }\end{array}$} & \multicolumn{4}{|c|}{$\begin{array}{c}\text { Method3 } \\
\text { (SAT) }\end{array}$} \\
\cline { 2 - 5 } & \#Var & \#Cl & \#Lit & Time \\
\hline $1,14,14,15$ & 2324 & 44034 & 440910 & 799.8 \\
\hline $1,9,9,10$ & 2398 & 11164 & 35570 & 138.6 \\
\hline $1,4,4,5$ & 2824 & 10890 & 30800 & 179.4 \\
\hline $1,1,1,2$ & 4714 & 14084 & 35168 & 369.3 \\
\hline
\end{tabular}

(b) Statistics for mul16

execution based algorithm. We should develop SAT-based manipulations of reachable states.

\section{ACKNOWLEDGMENTS}

We would like to express our thanks to Research Associate Takashi Horiyama, Qiang Zhu and other members of Watanabe Laboratory at Nara Institute of Science and Technology for their discussions and comments. We also thank to VDEC at the University of Tokyo for their support for the usage of CAD tools. The part of this work is supported by a Grant in Aid for Scientific Research from the Ministry of Education, Science, Sports and Culture, Specified Area Research 09245101 and 11480068, and a grant form Nikkei BP IP design award.

\section{REFERENCES}

[1] A. Biere, A. Cimatti, E. M. Clarke, M. Fujita, and Y. Zhu, "Symbolic Model Checking using SAT procedures instead of BDDs," Proc. of DAC'99, June 1999.

[2] K. Nakamura, K. Takagi, S. Kimura and K. Watanabe, "Waiting False Path Analysis of Sequential Logic Circuits for Performance Optimization," Proc. of ICCAD'98, pp. 392-395, Nov. 1998.

[3] K. Nakamura, S. Kimura, K. Takagi and K. Watanabe, "Timing Verification of Sequential Logic Circuits Based on Controlled Multi-clock Path Analysis," IEICE Transactions on Fundamentals, Vol. E81-A NO. 12, pp. 25152520, Dec. 1998.

[4] Anurag P. Gupta and Daniel P. Siewiorek, "Automated Multi-Cycle Symbolic Timing Verification of Microprocessor-based Designs," Proc. of 31st DAC, pp. 113-119, 1994

[5] Tracy Larrabee, "Test Pattern Generation Using Boolean Satisfiability," IEEE Transaction on Computer-Aided Design, pp. 4-15, Jan. 1992.

[6] H. Zhang, "SATO: An Efficient Propositional Prover," Proc. of International Conference on Automated Deduction'97, pp. 272-275, Jan. 1997.

[7] M. Davis and H. Putnam, "A Computing Procedure for Quantification Theory," Journal of the Association for Computing Machinery, 7, pp. 201-215, 1960.

[8] F. Brglez, D. Bryan, and K. Kozminski, "Combinational Profiles of Sequential Benchmark Circuits," IEEE 1989 International Symposium on Circuits and Systems Proceedings, pp. 1929-1934, May 1989.

[9] D. S. Johnson, and M. A. Trick, editors, "The second DIMACS implementation challenge," DIMACS Series in Discrete Mathematics and Theoretical Computer Science, 1993. (see http: //dimacs.rutgers.edu/Challenges/)

[10] L. G. Silva, J. P. M. Silva, L. M. Silveira, and K. A. Sakallah, "Timing Analysis Using Propositional Satisfiability," IEEE International Conference on Electronics, Circuits and Systems, Sep. 1998.

[11] O. Coudert, C. Berthet, and J. C Madre, "Verification of Sequential Machines Based on symbolic Execution," Automatic Verification Methods for Finite State Systems(LNCS 407), pp. 365-373, Sep. 1989.

[12] R. E. Bryant, "Symbolic Boolean Manipulation with Ordered Binary-Decision Diagrams," ACM Computing Surveys, vol. 24, no. 3, pp. 293-318, Sep. 1992.

[13] D. Plaisted and S. Greenbaum, "A Structure-Preserving Clause Form Translation," Journal of Symbolic Computation 2, pp. 293-304, 1986.

[14] P. C. McGeer, A. Saldanha, R. K. Brayton and A. Sangiovanni-Vincentelli, "Delay Models and Exact Timing Analysis," Logic Synthesis and Optimization, Kluwer Academic Publishers, pp. 167-189, 1993, ed. T. Sasao.

[15] J. Silva and K. Sakallah, "GRASP: A Search Algorithm for Propositional Satisfiability," IEEE Transactions on Computers, pp. 506-521, May, 1999.

[16] S. Yang, "Logic Synthesis and Optimization Benchmarks User Guide," Technical Report 1991-IWLS-UG-Saeyang, Jan. 1991. 